\title{
Coordinated Control for a Group of Interconnected Pairwise Subsystems
}

\author{
Chen Ma and Xue-Bo Chen \\ School of Electronics and Information Engineering, Liaoning University of Science and Technology, Anshan 114051, China \\ Correspondence should be addressed to Xue-Bo Chen; xuebochen@126.com
}

Received 21 June 2013; Accepted 29 August 2013

Academic Editor: Bo Shen

Copyright ( 12013 C. Ma and X.-B. Chen. This is an open access article distributed under the Creative Commons Attribution License, which permits unrestricted use, distribution, and reproduction in any medium, provided the original work is properly cited.

The implementation of pairwise decomposition is discussed on an interconnected system with uncertainties. Under the concept of system inclusion, two systems with the same expanded system achieved by the same expand transformation are considered as approximations. It is proven that a coordinated controller can be found to stabilize both the two systems. This controller is contracted from the coordinated controller of expanded system, with each pairwise subsystem having information structure constraint taken into consideration. At last, this controller design process is applied on a four-area power system treated as a group of subsystems with information structure constraints.

\section{Introduction}

Complex systems in real world are usually composed of a large group of interconnected subsystems. The interconnections among the subsystems are commonly presented in dynamics, and not only their weight values but also their connections with others keep evolving from time to time. Decentralized control is an ideal control strategy to handle the structural perturbations. Inclusion principle [1$3]$ is widely used as a general mathematical framework of decomposition, for example, automatic generation control (AGC) for a four-area power system [4-8], formation control of unmanned aerial vehicles [9], and structural vibration control of tall buildings under seismic excitations [10-12].

Particularly, pairwise decomposition provided in [4-8] can take full use of interconnections in the system, by treating each pair of subsystems with information structure constraint as a basic connected unit. Based on the inclusion principle framework, the system will be expanded into a much bigger space in a recurrent reverse order, so that the system is completely decomposed. Then a pairwise coordinated controller for the expanded system will be constructed by achieving coordinated consensus of each pairwise subsystem in parallel. After properly compensated, the controller can be contracted into the original space to fix the original system.
However, to apply pairwise decomposition methodology, an explicitly defined overall system model in particular superposition form is needed, and this condition may not always be satisfied due to system complexity. The work of this paper is to present an implementation approach of pairwise decomposition for interconnected system with state uncertainties. As the basis of system expansion and contraction, adequate knowledge of interconnection structure between pairwise subsystem is necessary, and this is the presumption to apply pairwise decomposition in this paper. For the system whose model is uncertain, the inclusion principle can not achieve its expansion exactly. But under the circumstance that the interconnection structure of system is available, an approximate expanded system can be constructed instead. Motivated by the idea that the whole system could achieve high performance only if each part could be consistent, an expanded system can be constructed, which comprises all pairwise subsystems with information structure constraints of the original system, and this expanded system is treated as an approximate expansion of the origin. According to the inclusion conditions, the expanded system can be contracted to the original space. A contraction dual to the expansion can always be found, so that the contracted system and original system are approximate in state dynamic. In this way, the coordinated controller that can stabilize the contracted 
system is also suitable for the original system. Similar to the system level, the coordinated controller of contracted system is also established by contracting from that of expanded system properly. In fact, this contracted controller can be used directly on the original system. As long as the dynamic of original system is adequately included in the expanded system, the contracted controller can be used as a suboptimal controller of the origin. The approximation of this paper mainly represents how good the expanded system would include the original system state. However, it is difficult to describe the approximation without a comparison of control performances. Considering the uncertainties of system state, a static state feedback controller at each subsystem is designed to robustly stabilize the system dynamics. This control design process mainly depends on the decentralized system form; it can suit a group of systems which can only use local information, for example, the multiagent system. Moreover, just the same as the ordinary pairwise decomposition, this process is also able to deal with the information structure constraints variation.

The organization of this paper is as follows. In the next section, preliminaries of permuted inclusion principle and system contraction are provided. The main result is presented in Section 2, where the approximate expansion under the concept of system inclusion is discussed, as well as the controller design procedure. In Section 3, a simulation example is provided to illustrate the proposed method on a group of subsystems with information structure constraints.

\section{Preliminaries}

The controller design process provided in this paper mainly relies on the permuted inclusion principle $[6,7]$ and system contraction $[7,13]$ that are presented in the following.

2.1. Permuted Inclusion Principle. Suppose that system $\overline{\mathbf{S}}$ is a group of interconnected subsystems and each subsystem $\overline{\mathbf{S}}_{i}$ is connected to every other counterparts. Then system $\overline{\mathbf{S}}$ can be decomposed into the expanded space of $N(N-1) / 2$ pairwise subsystems with a pair recurrent reverse order subscripts as follows:

$$
\begin{gathered}
\overline{\mathbf{S}}_{i j}: \overline{\mathbf{S}}_{12}, \overline{\mathbf{S}}_{23}, \overline{\mathbf{S}}_{13}, \overline{\mathbf{S}}_{34}, \overline{\mathbf{S}}_{24}, \overline{\mathbf{S}}_{14}, \ldots, \\
\overline{\mathbf{S}}_{(N-1) N}, \overline{\mathbf{S}}_{(N-2) N}, \ldots, \overline{\mathbf{S}}_{2 N}, \overline{\mathbf{S}}_{1 N} .
\end{gathered}
$$

Notice that the pairwise subsystems are arranged by a reverse order of subscript $j$, and this unnatural order enables the last one or some subsystems of the sequence to disconnect from, or connect to on the contrary, the overall system without impact on the remaining orders. It is convenient for representing the system information structure constraints variations.

The expected pairwise subsystems order is established by both row and column permutation matrices, which are composed of a series of basic permutation matrices representing a special case of nonsingular transformations. Assume that $I_{k}$ is a subidentity matrix corresponding to the subsystem $\overline{\mathbf{S}}_{k}$, as provided in $[6,7]$,

$$
\begin{aligned}
P & =\overleftrightarrow{\Pi}_{i=1}^{N-2} \overleftrightarrow{\Pi}_{j=1}^{N-i-1} \overleftrightarrow{\Pi}_{k=1+i(i-1)}^{N(N-j)-i(j+1)} p_{k(k+1)} \\
P^{-1} & =\overleftrightarrow{\Pi}_{i=1}^{N-2} \overleftrightarrow{\Pi}_{j=1}^{N-i-1} \overleftrightarrow{\Pi}_{\mathrm{k}=1+i(i-1)}^{N(N-j)-i(j+1)} p_{k(k+1)}^{T}
\end{aligned}
$$

where the signs " $\longleftarrow$ " and “ $\rightarrow$ " indicate right and left directional multiplying operations, and

$$
\begin{aligned}
p_{k(k+1)} & =\operatorname{blockdiag}\left(I_{1}, \ldots, I_{k-1},\left[\begin{array}{cc}
0 & I_{k} \\
I_{k+1} & 0
\end{array}\right], I_{k+2}, \ldots, I_{\widetilde{N}}\right), \\
p_{k(k+1)}^{-1} & =p_{k(k+1)}^{T} \\
& =\operatorname{blockdiag}\left(I_{1}, \ldots, I_{k-1},\left[\begin{array}{cc}
0 & I_{k+1} \\
I_{k} & 0
\end{array}\right], I_{k+2}, \ldots, I_{\widetilde{N}}\right)
\end{aligned}
$$

are the basic permutation matrices for the $k$ and $k+1$ groups of adjacent columns and rows, respectively. The $\widetilde{N}$ in (3) indicates the number of subsystems in the expanded system, and here $\widetilde{N}=N(N-1)$. The literature [8] provides an alternative matrix position-based form to construct this permutation matrix $P$ more simply. Use $P(m, n)_{b}$ to notate the block position in $P$ of subidentity matrices $I_{i}$ and $I_{j}$ corresponding to pairwise subsystem $\overline{\mathbf{S}}_{i j}$ in $P$; then it comes

$$
\begin{array}{r}
P((N i-j+1),[j(j-1)-2(i-1)-1])_{b}=I_{i}, \\
P([N(j-1)-i+1],[j(j-1)-2(i-1)])_{b}=I_{j}, \\
i, j=1,2, \ldots, k, \ldots, N, \quad i \neq j .
\end{array}
$$

Example 1. Consider an expansion for system $\overline{\mathbf{S}}$ with full network structure and $N=3$; its pairwise subsystems can be ordered as

$$
\overline{\mathbf{S}}_{i j}: \overline{\mathbf{S}}_{12}, \overline{\mathbf{S}}_{23}, \overline{\mathbf{S}}_{13}
$$

According to (4), the block positions of $I_{i}$ and $I_{j}$ to $\overline{\mathbf{S}}_{i j}$ can be obtained as

$$
\begin{array}{ll}
\overline{\mathbf{S}}_{12}: P(2,1)_{b}=I_{1}, & P(3,2)_{b}=I_{2}, \\
\overline{\mathbf{S}}_{23}: P(4,3)_{b}=I_{2}, & P(5,4)_{b}=I_{3}, \\
\overline{\mathbf{S}}_{13}: P(1,5)_{b}=I_{1}, & P(6,6)_{b}=I_{3} .
\end{array}
$$

that is to say

$$
P=\left[\begin{array}{cccccc}
0 & 0 & 0 & 0 & I_{1} & 0 \\
I_{1} & 0 & 0 & 0 & 0 & 0 \\
0 & I_{2} & 0 & 0 & 0 & 0 \\
0 & 0 & I_{2} & 0 & 0 & 0 \\
0 & 0 & 0 & I_{3} & 0 & 0 \\
0 & 0 & 0 & 0 & 0 & I_{3}
\end{array}\right]
$$

It is equivalent to the result of (2) when $N=3$. 
Consider an interconnected system $\overline{\mathbf{S}}$ in compacted form and its expanded system $\widetilde{\mathbf{S}}_{P}$ as follows:

$$
\begin{gathered}
\overline{\mathbf{s}}: \dot{\bar{x}}=\bar{A} \bar{x}+\bar{B} \bar{u}, \quad \bar{y}=\bar{C} \bar{x}, \\
\widetilde{\mathbf{S}}_{P}: \dot{\bar{x}}_{P}=\widetilde{A}_{P} \widetilde{x}_{P}+\widetilde{B}_{P} \widetilde{u}_{P}, \quad \widetilde{y}_{P}=\widetilde{C}_{P} \widetilde{x}_{P},
\end{gathered}
$$

where $\bar{x}(t) \in \mathbf{R}^{\bar{n}}, \bar{u}(t) \in \mathbf{R}^{\bar{m}}$, and $\bar{y}(t) \in \mathbf{R}^{\bar{l}}$ are the state, input, and output vectors of the system $\overline{\mathbf{S}} ; \widetilde{x}_{P}(t) \in \mathbf{R}^{\widetilde{n}}, \widetilde{u}_{P}(t) \in \mathbf{R}^{\widetilde{m}}$, and $\tilde{y}_{P}(t) \in \mathbf{R}^{\tilde{l}}$ are those of system $\widetilde{\mathbf{S}}_{P}$. It is supposed that $\bar{n} \leq \widetilde{n}, \bar{m} \leq \widetilde{m}$, and $\bar{l} \leq \tilde{l}$.

For the input-state-output inclusion principle mentioned in $[14,15]$, a definition of the permuted inclusion principle is given.

Definition 2. The system $\widetilde{\mathbf{S}}_{P}$ includes the system $\overline{\mathbf{S}}$, or $\widetilde{\mathbf{S}}_{P} \supset \overline{\mathbf{S}}$, if there exists a quadruplet of full rank matrices $\left\{V_{P}, U_{P}, Q_{P}, S_{P}\right\}$ satisfying $U_{P} V_{P}=I_{n}$, such that for any $\bar{x}_{0} \in \mathbf{R}^{\bar{n}}$ and any $\bar{u} \in \mathbf{R}^{\bar{m}}$, the conditions $\tilde{x}_{P 0}=V_{P} \bar{x}_{0}$ and $\bar{u}=Q_{P} \widetilde{u}_{P}$ imply $\bar{x}\left(t ; t_{0}, \bar{x}_{0}, \bar{u}\right)=U_{P} \widetilde{x}_{P}\left(t ; t_{0}, \widetilde{x}_{P 0}, \widetilde{u}_{P}\right)$ and $\bar{y}[\bar{x}(t)]=S_{P} \tilde{y}_{P}\left[\tilde{x}_{P}(t)\right]$ for all $t \geq t_{0}$.

Call the system $\overline{\mathbf{S}}$ a contraction of system $\widetilde{\mathbf{S}}_{P}$. It is supported by the inclusion principle that all information about the behavior of $\overline{\mathbf{S}}$ is included in $\widetilde{\mathbf{S}}_{P}$, such as stability and optimality. One of the necessary and sufficient conditions for the inclusion is restriction, the following theorem considers the restriction type (d) $([2,3,7])$.

Theorem 3. The system $\overline{\mathbf{S}}$ is a typical restriction of the system $\widetilde{\mathbf{S}}_{P}$, if there is a triplet of full rank matrices $\left\{V_{P}, Q_{P}, S_{P}\right\}$ such that

$$
V_{P} \bar{A}=\widetilde{A}_{P} V_{P}, \quad V_{P} \bar{B} Q_{P}=\widetilde{B}_{P}, \quad \bar{C}=S_{P} \widetilde{C}_{P} V_{P} .
$$

Proof. The proof follows directly from the results in $[6,7,14$, 15].

The systems $\overline{\mathbf{S}}$ and $\widetilde{\mathbf{S}}_{P}$ are related by

$$
\begin{gathered}
\widetilde{A}_{P}=V_{P} \bar{A} U_{P}+M_{A}^{P}, \widetilde{B}_{P}=V_{P} \bar{B} Q_{P}+M_{B}^{P}, \\
\widetilde{C}_{P}=T_{P} \bar{C} U_{P}+M_{C}^{P},
\end{gathered}
$$

where $M_{A}^{P}, M_{B}^{P}$, and $M_{C}^{P}$ are complementary matrices with proper dimensions. See $[6,7]$ for details.

2.2. System Contraction. One of the difficulties in applying system contraction by inclusion principle is that the conditions may be too restrictive, and a complete contraction from the given expanded system $\widetilde{\mathbf{S}}_{P}$ to system $\overline{\mathbf{S}}$ will not always exist. It is indicated by the restriction conditions of (9) that system $\widetilde{\mathbf{S}}_{P}$ completely includes $\overline{\mathbf{S}}$ if and only if it is uncontrollable. A natural way to resolve this problem is to introduce an incomplete contraction as an approximation. Split the permuted state matrix $\widetilde{A}_{P}$ into two parts as

$$
\widetilde{A}_{P}=\widetilde{A}_{R P}+\widetilde{M}_{A P},
$$

where $\widetilde{A}_{R P}$ is the part that can be contracted as (8) implies, $\widetilde{M}_{A P}$ is a complementary matrix with proper dimension standing for the remnant after contraction from the expanded space. System $\overline{\mathbf{S}}$ is a reduced-order model of system $\widetilde{\mathbf{S}}_{P}$, according to the restriction conditions in (9) and (10), and take the state matrix for example, this incomplete system contraction requires that

$$
V_{P} \bar{A}=\left(\widetilde{A}_{P}-\widetilde{M}_{A P}\right) V_{P} .
$$

There are arbitrary choices of the expanding transformation matrix $V_{P}$. Since this paper is based on the pairwise decomposition methodology, $V_{P}$ is chosen as the same form of that in $[6,7]$, which will be presented in next section. Anyway, when $V_{P}$ is confirmed according to the inclusion condition, here goes

$$
\widetilde{M}_{A P} V_{P}=\widetilde{A}_{P} V_{P}-V_{P} \bar{A} .
$$

To satisfy the restriction condition, there must be

$$
\bar{A}=\left(V_{P}^{T} V_{P}\right)^{-1} V_{P}^{T} \widetilde{A}_{P} V_{P}
$$

so that $\left\|\widetilde{M}_{A P} V_{P}\right\|$ will be minimum, and this results in the minimal norm solution

$$
\widetilde{M}_{A P}=\left[I-V_{P}\left(V_{P}^{T} V_{P}\right)^{-1} V_{P}^{T}\right] \widetilde{A}_{P} V_{P}\left(V_{P}^{T} V_{P}\right)^{-1} V_{P}^{T} .
$$

\section{Pairwise Decomposition for a Group of Interconnected Subsystems}

Assume that system $\mathbf{S}$ is composed of a group of interconnected subsystems as the coordinated control target,

$$
\begin{aligned}
\mathbf{S}=\left\{\mathbf{S}_{i}\right\}: \dot{x}=A x+B u, \quad y=C x, & \\
\mathbf{S}_{i}: \dot{x}_{i}= & A_{i i} x_{i}+B_{i i} u_{i} \\
& +\sum_{\substack{j=1 \\
j \neq i}}^{N} e_{i j}(t) A_{i j} x_{j}, \quad y_{i}=C_{i i} x_{i},
\end{aligned}
$$

where $x_{i}(t) \in \mathbf{R}^{n_{i}}, u_{i}(t) \in \mathbf{R}^{m_{i}}$, and $y_{i}(t) \in \mathbf{R}^{l_{i}}$ are the state, input, and output vectors of $\mathbf{S}_{i}$ at time $t \in \mathbf{R}$ system matrices $A, B$, and $C$ are compacted forms of $A_{i j}, B_{i i}$, and $C_{i i}$ in proper dimensions, respectively. Notations $x_{i}\left(t ; t_{0}, x_{i 0}, u_{i}\right)$ and $y_{i}\left[x_{i}(t)\right]$ denote unique solutions of $\mathbf{S}_{i}$ for the initial time $t_{0}$, the initial state vector $x_{i 0}$, and a fixed control input $u_{i}, e_{i j}(t)$ is element of the interconnection matrix $E=\left(e_{i j}\right) \in \mathbf{R}^{N \times N}$. Under the concept of pairwise decomposition, if at least one of interconnections $e_{i j}(t) \neq 0$ or $e_{j i}(t) \neq 0$, then it appears that subsystems $\mathbf{S}_{i}$ and $\mathbf{S}_{j}$ are connected. Call

$$
\mathbf{S}_{i j}: \begin{cases}\dot{x}_{i}=A_{i i} x_{i}+e_{i j}(t) A_{i j} x_{j}+B_{i i} u_{i}, & y_{i}=C_{i i} x_{i} \\ \dot{x}_{j}=A_{j j} x_{j}+e_{j i}(t) A_{j i} x_{i}+B_{j j} u_{j}, & y_{j}=C_{j j} x_{j} \\ & i, j=1,2, \ldots, N, \\ & i \neq j\end{cases}
$$

a pairwise subsystem with basic interconnection. 
The time-varying parameters $e_{i j}(t)$ and $e_{j i}(t)$ in (17) can describe the dynamic weight values between the connected subsystems $\mathbf{S}_{i}$ and $\mathbf{S}_{j}$. They represent the information structure constraints of the interconnected system and play a very important role in the system dynamic. In the literature [16], a fundamental interconnection (adjacency) matrix $\bar{E}=\left(\bar{e}_{i j}\right) \epsilon$ $\mathbf{R}^{N \times N}$ is defined in order to describe the normal structure of a given system graph. This notation can also be used here to indicate whether there is information structure constraint between a subsystem pair, by the rule

$$
\bar{e}_{i j}=\left\{\begin{array}{ll}
1, & e_{i j}(t) \neq 0 \\
0, & e_{i j}(t)=0,
\end{array} \quad \bar{e}_{i i}=0 .\right.
$$

When $\bar{e}_{i j}=1$, it indicates that there is interconnection from subsystem $\mathbf{S}_{i}$ to $\mathbf{S}_{j}$, and $\bar{e}_{i j}=0$ indicates not. This binary interconnection matrix $\bar{E}$ will be used later in the inclusion principle framework. If one of $\bar{e}_{i j}$ and $\bar{e}_{j i}$ is equal to 0 , the pairwise subsystem $\mathbf{S}_{i j}$ is half connected; the original information structures of $\mathbf{S}_{i j}$ would be changed by using the coordinated control mentioned earlier. In this case, the sequential LQ optimization provided in $[3,17]$ can be consulted to keep the information structure of $\mathbf{S}_{i j}$. Moreover, note that $\bar{e}_{i j}$ and $\bar{e}_{j i}$ will both be valued 0 under some circumstances, which means that the pairwise subsystem $\mathbf{S}_{i j}$ will be disjointed. The disconnected modes have been discussed in [6,7]. Particularly, when $\bar{e}_{i j}$ and $\bar{e}_{j i}$ evolute in a dynamical way and enforce $S_{i j}$ to disjoint and then joint again, the discussion is provided in [8].

Theoretically, any existing control technique can be applied to the coordinated control of this pairwise subsystem $\mathbf{S}_{i j}$. Take pairwise subsystem $\mathbf{S}_{i j}$ as this compact form

$$
\mathbf{S}_{i j}: \dot{x}_{i j}=A_{D i j} x_{i j}+B_{D i j} u_{i j}, \quad y_{i j}=C_{D i j} x_{i j},
$$

with $x_{i j}=\left[x_{i}, x_{j}\right]^{T}, u_{i j}=\left[u_{i}, u_{j}\right]^{T}$, and $y_{i j}=\left[y_{i}, y_{j}\right]^{T}$, and

$$
\begin{gathered}
A_{D i j}=\left[\begin{array}{cc}
A_{i i} & e_{i j}(t) A_{i j} \\
e_{j i}(t) A_{j i} & A_{j j}
\end{array}\right], \\
B_{D i j}=\left[\begin{array}{cc}
B_{i i} & 0 \\
0 & B_{j j}
\end{array}\right], \\
C_{D i j}=\left[\begin{array}{cc}
C_{i i} & 0 \\
0 & C_{j j}
\end{array}\right] .
\end{gathered}
$$

Call

$$
\mathrm{C}_{i j}: u_{i j}=-K_{D i j} x_{i j}
$$

the basic coordinated controller, if it can stabilize the closed loop pairwise subsystem

$$
\mathbf{S}_{i j}^{C}: \dot{x}_{i j}=\left(A_{D i j}-B_{D i j} K_{D i j}\right) x_{i j}, \quad y_{i j}=C_{D i j} x_{i j} .
$$

For every pair of subsystems $\mathbf{S}_{i j}$ with information structure constraints $i=j-k, j=2,3, \ldots, N, k=1,2, \ldots, j-1$, their basic coordinated controllers can be constructed in this way.
As the fundamental idea of pairwise decomposition, a given system should be expanded following the recurrent reverse order first, so that a coordinated controller can be designed to stabilize all of the pairwise subsystems and then contracted to the original space. However, restricted by the mathematical framework of inclusion principle, it is difficult to expand the system with uncertainties in its dynamics. Consider the procedure of pairwise decomposition, the original states can be almost included in the block-diagonal expanded system which is composed of state functions of all pairwise subsystems,

$$
\begin{gathered}
\widetilde{\mathbf{S}}_{P D}=\left\{\mathbf{S}_{12}, \mathbf{S}_{23}, \mathbf{S}_{13}, \mathbf{S}_{34}, \mathbf{S}_{24}, \mathbf{S}_{14}, \ldots, \mathbf{S}_{2 N}, \mathbf{S}_{1 N}\right\}: \\
\dot{\tilde{x}}_{P}=\widetilde{A}_{P D} \widetilde{x}_{P}+\widetilde{B}_{P D} \widetilde{u}_{P}, \quad \widetilde{y}_{P}=\widetilde{C}_{P D} \widetilde{x}_{P}, \\
\widetilde{A}_{P D}=\operatorname{blockdiag}\left(A_{D i j}\right), \\
\widetilde{B}_{P D}=\operatorname{blockdiag}\left(B_{D i j}\right), \\
\widetilde{C}_{P D}=\operatorname{blockdiag}\left(C_{D i j}\right) .
\end{gathered}
$$

This block-diagonal system is a reasonable approximate expansion of the origin. To achieve this form, the interconnection structure of system $\mathbf{S}$ should be available, and this is also the restriction in using inclusion principle. The interconnection structure is supposed to be given by the fundamental interconnection matrix $\bar{E}=\left(\bar{e}_{i j}\right)$. By expanding the original space of system $\mathbf{S}$ into a bigger space of system $\widetilde{\mathbf{S}}_{P D}$ in recurrent reverse order, take the state matrix as an example, the transformation matrices of pairwise decomposition can be selected as

$$
\begin{gathered}
V=\operatorname{blockdiag}(\overbrace{I_{n_{1}} I_{n_{1}} \cdots I_{n_{1}}}^{\sum_{k=1}^{N} \bar{e}_{1 k}}, \ldots, \overbrace{I_{n_{N}} I_{n_{N}} \cdots I_{n_{N}}}^{\sum_{k=1}^{N} \bar{e}_{N k}})^{T}, \\
U=\operatorname{blockdiag}\left(\frac{1}{\sum_{k=1}^{N} \bar{e}_{1 k}}[\overbrace{I_{n_{1}} \cdots I_{n_{1}}^{N}}^{\sum_{k=1} \bar{e}_{1 k}}], \ldots,\right. \\
\left.\frac{1}{\sum_{k=1}^{N} \bar{e}_{N k}}[\overbrace{I_{n_{N}} \cdots I_{n_{N}}}^{\sum_{k=1}^{N} \bar{e}_{N k}}]\right),
\end{gathered}
$$

$R, Q, T$, and $S$ have the same structure as their counterparts, respectively. Notice that there are arbitrary choices of these transformation matrices, and their forms are bound up with the inclusion form. Since the structure of expanded system $\widetilde{\mathbf{S}}_{P D}$ is confirmed, then the transformation matrices are also fixed, just as (24).

Consider the permuted inclusion principle; the transformation matrices will be permuted as

$$
\begin{array}{ll}
V_{P}=P_{A}^{-1} V, & U_{P}=U P_{A}, \\
R_{P}=P_{B}^{-1} R, & Q_{P}=Q P_{B}, \\
T_{P}=P_{C}^{-1} T, & S_{P}=S P_{C} .
\end{array}
$$


Therefore the state matrices of systems $\mathbf{S}$ and $\widetilde{\mathbf{S}}_{P D}$ are related by (12), and the relationship of the state, input, and output vectors can be obtained by Definition 2 as

$$
x=U_{P} \tilde{x}_{P}, \quad u=Q_{P} \widetilde{u}_{P}, \quad y=S_{P} \tilde{y}_{P} .
$$

At the same time, a virtual system $\overline{\mathbf{S}}$ can be constructed as another contraction of system $\widetilde{\mathbf{S}}_{P D}$. The state dynamic of system $\overline{\mathbf{S}}$ is in a certain form, and $\overline{\mathbf{S}}$ is raised as an estimation of the original system $\mathbf{S}$. According to the contraction condition, it is possible to use the transformation matrix $V_{P}$ such that system $\widetilde{\mathbf{S}}_{P D}$ can be contracted to $\overline{\mathbf{S}}$ by (12) after an appropriate compensation. This process will also lead to the same relationship as (26). In this way, systems $\mathbf{S}$ and $\overline{\mathbf{S}}$ may share the same state, input, and output vectors, since they have the same expanded system $\widetilde{\mathbf{S}}_{P D}$ which is calculated by the same transformation matrices. It can be concluded that systems $\mathbf{S}$ and $\overline{\mathbf{S}}$ represent a pair of systems with approximate dynamics, and the bias between them is mainly reflected in compensation $\widetilde{M}_{A P}$ of the contraction procedure.

Suppose that the expanded system $\widetilde{\mathbf{S}}_{P D}$ comprises every state function of pairwise subsystem $\mathbf{S}_{i j}$ in system $\mathbf{S}$, and the pairwise subsystems are arranged in the recurrent reverse order as (23). Each $\mathbf{S}_{i j}$ is stabilized by the basic coordinated controller (21); then the coordinated controller for $\widetilde{\mathbf{S}}_{P D}$ can be constructed in a block-diagonal form as

$$
\widetilde{\mathbf{C}}_{P D}=\left(\mathbf{C}_{i j}\right): \widetilde{u}_{P}=-\widetilde{K}_{D} \widetilde{x}_{P}=-\operatorname{blockdiag}\left(K_{D i j}\right) \tilde{x}_{P} .
$$

It is clear that a redundant control set is established with all pairwise controllers, which contains all necessary coordinated information $K_{D i j}$ for both system $\mathbf{S}$ and $\overline{\mathbf{S}}$. When the structure form of the estimator $\overline{\mathbf{S}}$ is determined, the coordinated controller of system $\mathbf{S}$ can be obtained by contracting $\widetilde{K}_{D}$ together with a proper compensator $\widetilde{M}_{K P}$. The contraction is checked by the following theorem.

Theorem 4. For the systems mentioned above, system $\widetilde{\mathbf{S}}_{P D}$ is the expansion for both systems $\mathbf{S}$ and $\overline{\mathbf{S}}$. The state feedback controller $C: u=-K x$ can stabilize the closed loop system of $\mathbf{S}$, if the controller $\bar{C}$ of system $\overline{\mathbf{S}}$ can be contracted from $\widetilde{C}_{P}$, and it satisfies

$$
K=\bar{K}=Q_{P}\left(\widetilde{K}_{D}+\widetilde{M}_{K P}\right) V_{P}
$$

Proof. Since system $\overline{\mathbf{S}}$ is a contraction system $\widetilde{\mathbf{S}}_{P D}$, supported by the contraction condition (12), the state function of system $\widetilde{\mathbf{S}}_{P D}$ is rewritten as

$$
\dot{\tilde{x}}_{P}=\left(\widetilde{A}_{P D}-\widetilde{M}_{A P}\right) \widetilde{x}_{P}+\widetilde{B}_{P D} \widetilde{u}_{P},
$$

and it apparently indicates the controller form of (5). According to the inclusion principle, here goes

$$
x=U_{P} \tilde{x}_{P}, \quad u=Q_{P} \tilde{u}_{P} .
$$

moreover, the approximation between systems $\mathbf{S}$ and $\overline{\mathbf{S}}$ may indicate that $x=\bar{x}$ and $u=\bar{u}$, so that the controllers of systems $\mathbf{S}$ and $\widetilde{\mathbf{S}}_{P D}$ are related as

$$
u=-K x, \quad \widetilde{u}_{P}=-\left(\widetilde{K}_{D}+\widetilde{M}_{K P}\right) \tilde{x}_{P} .
$$

Notice that $U_{P} V_{P}=I_{n}$, then (30) and (31) will conclude that $K=Q_{P}\left(\widetilde{K}_{D}+\widetilde{M}_{K P}\right) V_{P}$.

Remark 5. The literature [6] provides a sufficient condition of connective stability. But since far more information might be accumulated in the largest singular values of subsystem matrices, the criterion of connective stability might be somewhat conservative.

The virtual system $\overline{\mathbf{S}}$ is used as the estimator of system $\mathbf{S}$, and it may have many possible forms. This diversity will mainly impact on the controller design process in determining the compensator $\widetilde{M}_{K P}$. One of the most challenging problems in controller design for multiagent systems is the estimation of information structures among agents. The further research of this paper on implementation of pairwise decomposition in systems with dynamic information structure constraints, as well as the estimation of the interconnection structure, is undergoing. This issue is based on inclusion principle for time-varying system ([18]) and method in dealing with the structure perturbation under the concept of pairwise decomposition ([8]). However, in a particular case when the state function of each subsystem satisfies the linear superposition principle, there is a way to determine the structure of $\widetilde{M}_{K P}$ much more easily.

Consider the mathematical framework of permuted inclusion principle; this position information can be concluded with (8) by using the block row-order of sub-identity matrices. Consider that system $\mathbf{S}$ is in full network structure, the row-order of a particular pairwise subsystem $S_{i j}$ is

$$
c_{i}=j(j-1)-2(i-1)-1, \quad c_{j}=j(j-1)-2(i-1) .
$$

Besides, the row-order of every pairwise subsystem can also be concluded in this way as

$$
\begin{gathered}
c_{i k_{i}}= \begin{cases}k_{i}\left(k_{i}-1\right)-2(i-1)-1, & i<k_{i} \\
i(i-1)-2\left(k_{i}-1\right), & i>k_{i}\end{cases} \\
c_{j k_{j}}= \begin{cases}k_{j}\left(k_{j}-1\right)-2(j-1)-1, & j<k_{j} \\
j(j-1)-2\left(k_{j}-1\right), & j>k_{j}\end{cases} \\
i, j=1,2, \ldots, N, \quad k_{i}, k_{j}=1,2, \ldots, N, \quad i \neq j, \quad k_{i}, k_{j} \neq i, j .
\end{gathered}
$$

so that the complementary matrix $\widetilde{M}_{K P}$ can be constructed by the following lemma.

Lemma 6. Suppose that system $\mathbf{S}$ is in full network structure; the row-order of subsystems in each pairwise subsystem is concluded as (32) and (33), and $\widetilde{M}_{K P}$ complements the information structure constraints bias between system $\mathbf{S}$ and system 
$\overline{\mathbf{S}}$. Then $\widetilde{M}_{K P}$ can be presented by the information structure of corresponding pairwise subsystem $\mathbf{S}_{i j}$ as

$$
\begin{aligned}
& \widetilde{M}_{K P}\left(c_{i k_{i}}, c_{j}\right)_{b}=K_{i j}, \quad \widetilde{M}_{K P}\left(c_{j k_{j}}, c_{i}\right)_{b}=K_{j i} \\
& i, j=1,2, \ldots, N, \quad k_{i}, k_{j}=1,2, \ldots, N, \quad i \neq j, \quad k_{i}, k_{j} \neq i, j .
\end{aligned}
$$

This matrix structure-based lemma is convenient for calculation, especially for real-time control in practice.

Example 7. Also consider system $\mathbf{S}$ with $N=3$ subsystems, and its recurrent reverse order is presented as (5). According to (32) and (33), the calculation is proceeded as

$$
\begin{aligned}
& \mathrm{S}_{12}: c_{i}=1, \quad c_{j}=2, \\
& k_{i}=k_{j}=3, \quad c_{i k_{i}}=5, \quad c_{j k_{j}}=3, \\
& \Longrightarrow \widetilde{M}_{K P}(5,2)_{b}=K_{12}, \quad \widetilde{M}_{K P}(3,1)_{b}=K_{21} \\
& \mathrm{~S}_{23}: c_{i}=3, \quad c_{j}=4, \\
& k_{i}=k_{j}=1, \quad c_{i k_{i}}=2, \quad c_{j k_{j}}=6, \\
& \Longrightarrow \widetilde{M}_{K P}(2,4)_{b}=K_{23}, \quad \widetilde{M}_{K P}(6,3)_{b}=K_{32} \\
& \mathrm{~S}_{13}: c_{i}=5, \quad c_{j}=6, \\
& k_{i}=k_{j}=2, \quad c_{i k_{i}}=1, \quad c_{j k_{j}}=4, \\
& \Longrightarrow \widetilde{M}_{K P}(1,6)_{b}=K_{13}, \quad \widetilde{M}_{K P}(4,5)_{b}=K_{31} .
\end{aligned}
$$

So that the matrix $\widetilde{M}_{K P}$ can be constructed by Lemma 6 :

$$
\widetilde{M}_{K P}=\left[\begin{array}{cccccc}
0 & 0 & 0 & 0 & 0 & K_{13} \\
0 & 0 & 0 & K_{23} & 0 & 0 \\
K_{21} & 0 & 0 & 0 & 0 & 0 \\
0 & 0 & 0 & 0 & K_{31} & 0 \\
0 & K_{12} & 0 & 0 & 0 & 0 \\
0 & 0 & K_{32} & 0 & 0 & 0
\end{array}\right]
$$

\section{Automatic Generation Control (AGC) for a Four-Area Power System}

A four-area power system is shown in Figure 1; assume that areas 1,2 , and 3 contain three reheat turbine type thermal units and area 4 contains a hydro unit, respectively. Each pairwise subsystem is interconnected by tie line indicated by solid lines, and its information structure constraint is indicated by dotted ellipse. Details of the system description can be found in $[19,20]$. References [4-8] implement the pairwise decomposition methodology in the procedure of coordinated control to this four-area power system AGC. As a counterpart, the controller design procedure of the new pairwise decomposition modality in this paper is presented here. Consider the system dynamic bias between system $\mathbf{S}$ and system $\overline{\mathbf{S}}$ taken as approximation; each pairwise subsystem is robustly stabilized in terms of linear matrix inequalities (LMI) ([21, 22]).

Suppose that the system graph is undirected and $e_{i j}(t)=$ $e_{j i}(t)=1$ for description convenience. The pairwise subsystem model is provided in (17) as

$$
\begin{aligned}
\mathbf{S}_{i j}:\left[\begin{array}{c}
\dot{x}_{i} \\
\dot{x}_{j}
\end{array}\right]= & {\left[\begin{array}{cc}
A_{i i} & A_{i j} \\
A_{j i} & A_{j j}
\end{array}\right]\left(\left[\begin{array}{c}
x_{i} \\
x_{j}
\end{array}\right]+\left[\begin{array}{c}
w_{i} \\
w_{j}
\end{array}\right]\right) } \\
& +\left[\begin{array}{cc}
B_{i i} & 0 \\
0 & B_{j j}
\end{array}\right]\left[\begin{array}{c}
u_{i} \\
u_{j}
\end{array}\right]+\left[\begin{array}{cc}
G_{i i} & 0 \\
0 & G_{j j}
\end{array}\right]\left[\begin{array}{l}
\xi_{i} \\
\xi_{j}
\end{array}\right], \\
& {\left[\begin{array}{c}
y_{i} \\
y_{j}
\end{array}\right]=\left[\begin{array}{cc}
C_{i i} & 0 \\
0 & C_{j j}
\end{array}\right]\left[\begin{array}{c}
x_{i} \\
x_{j}
\end{array}\right] } \\
& i, j=1,2, \ldots, N, \quad i \neq j,
\end{aligned}
$$

where $x_{i j}=\left[x_{i}^{T}, x_{j}^{T}\right]^{T}, u_{i j}=\left[u_{i}^{T}, u_{j}^{T}\right]^{T}, \xi_{i j}=\left[\xi_{i}^{T}, \xi_{j}^{T}\right]^{T}$, and $y_{i j}=\left[y_{i}^{T}, y_{j}^{T}\right]^{T}$ are the state, control input, disturbance, and output vectors, respectively. $w_{i j}=\left[w_{i}^{T}, w_{j}^{T}\right]^{T}$ is the unmodeled or uncertain state dynamic. $A_{D i j}=\left(A_{i j}\right), B_{D i j}=$ $\left(B_{i i}\right), G_{D i j}=\left(G_{i i}\right)$, and $C_{D i j}=\left(C_{i i}\right)$ are system matrices with proper dimensions, respectively. The numerical values of the system matrices are given by

$$
A_{11}=\left[\begin{array}{cccccc}
-0.05 & 6 & 0 & 0 & 0 & -6 \\
0 & -0.1 & -1.01 & 1.11 & 0 & 0 \\
0 & 0 & -3.33 & 3.33 & 0 & 0 \\
-2.08 & 0 & 0 & -5 & 5 & 0 \\
-0.255 & 0 & 0 & 0 & 0 & -0.06 \\
1.33 & 0 & 0 & 0 & 0 & 0
\end{array}\right]
$$

$$
\begin{gathered}
A_{12}=\left[\begin{array}{cc}
0_{5 \times 1} & 0_{5 \times 5} \\
-0.444 & 0_{1 \times 5}
\end{array}\right], \quad A_{13}=\left[\begin{array}{ccc}
0_{4 \times 1} & 0_{4 \times 4} & 0_{4 \times 1} \\
0 & 0_{1 \times 4} & 0.06 \\
-0.444 & 0_{1 \times 4} & 0
\end{array}\right], \\
A_{14}=A_{21}=A_{32}=A_{13}, \quad A_{23}=\mathrm{A}_{31}=A_{41}=A_{12},
\end{gathered}
$$

$A_{44}$

$=\left[\begin{array}{cccccc}-0.0769 & 6.15 & 0 & 0 & 0 & -6.15 \\ 8.78 \times 10^{-4} & -2 & 2.2 & -0.198 & 2.11 \times 10^{-3} & 0 \\ -4.39 \times 10^{-4} & 0 & -0.1 & 0.0989 & 1.05 \times 10^{-3} & 0 \\ -8.56 \times 10^{-3} & 0 & 0 & -0.0205 & 0.0205 & 0 \\ -0.0255 & 0 & 0 & 0 & 0 & -0.06 \\ 0.444 & 0 & 0 & 0 & 0 & 0\end{array}\right]$,

$$
\begin{gathered}
B_{11}=B_{22}=B_{33}=\left[\begin{array}{llllll}
0 & 0 & 0 & 5 & 0 & 0
\end{array}\right]^{T}, \\
B_{44}=\left[\begin{array}{llllll}
0 & 0 & 0 & 0.0205 & 0 & 0
\end{array}\right]^{T}, \\
G_{11}=G_{22}=G_{33}=\left[\begin{array}{llllll}
-6 & 0 & 0 & 0 & 0 & 0
\end{array}\right]^{T}, \\
G_{44}=\left[\begin{array}{llllll}
-6.15 & 0 & 0 & 0 & 0 & 0
\end{array}\right]^{T}, \\
C_{11}=C_{22}=C_{33}=C_{44}=I_{6} .
\end{gathered}
$$




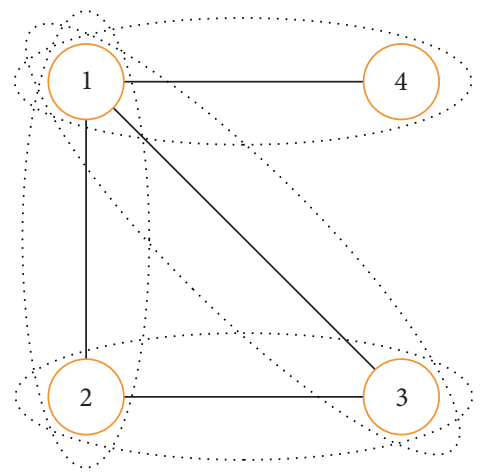

FIGURE 1: Schematic diagram of the four-area power system.

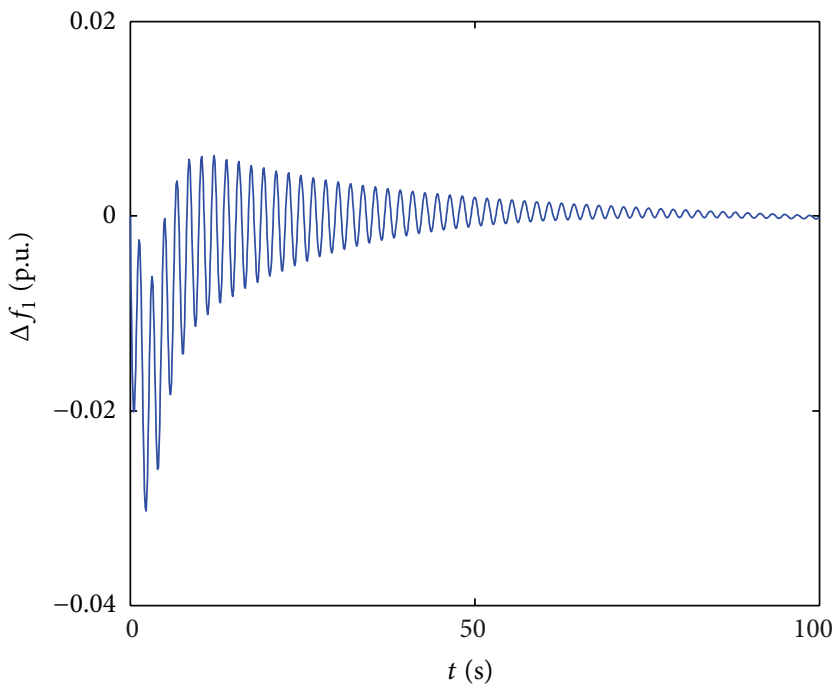

(a)

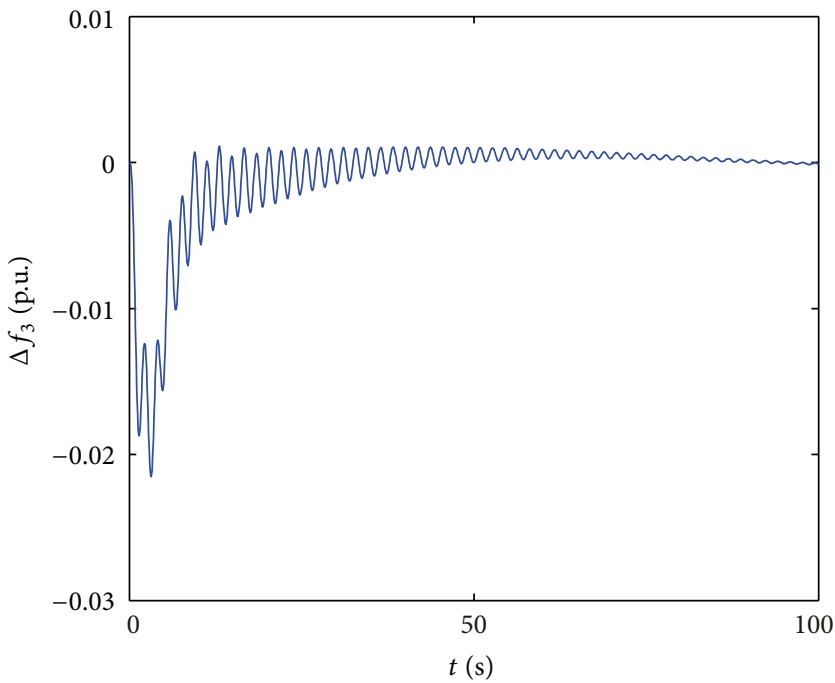

(c)

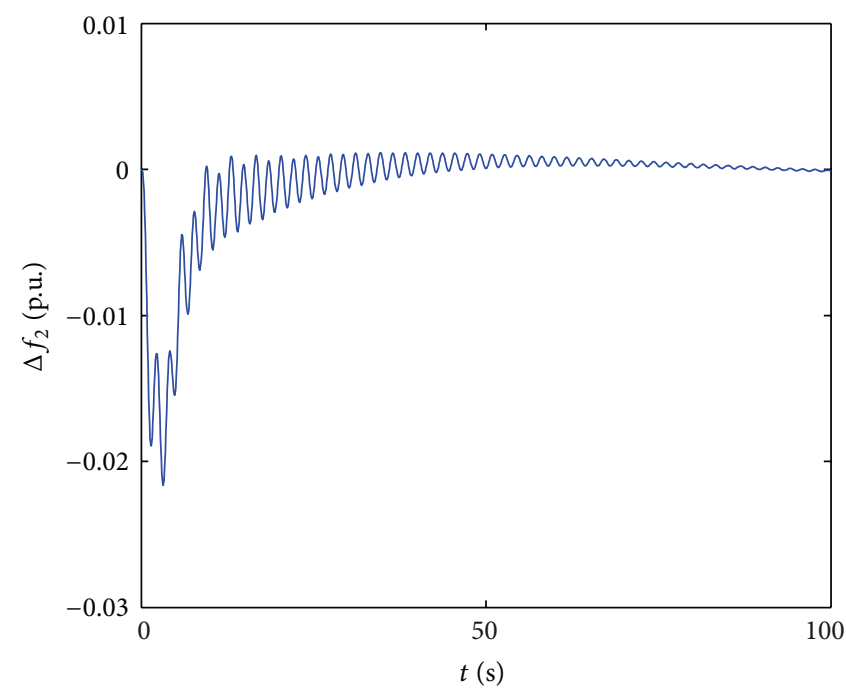

(b)

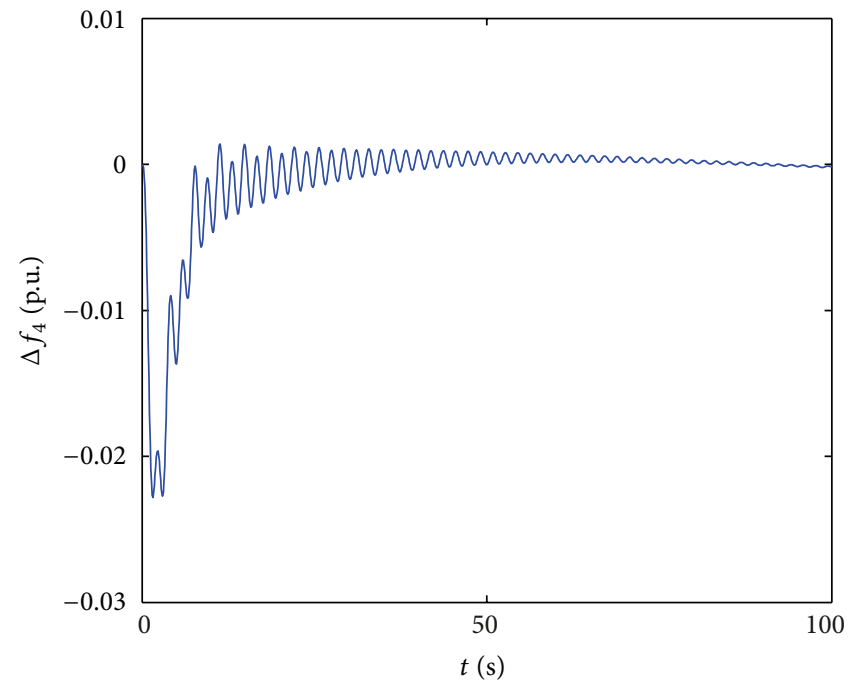

(d)

FIgURE 2: Deviations of frequency among the power system. 
$A_{22}$ and $A_{33}$ are the same as $A_{11}$ except that $A_{22}(6,1)=$ $A_{33}(6,1)=0.888$, respectively.

The quadratic constraints are imposed on the state uncertainty $w_{i j}$ as follows:

$$
w_{i j}^{T} w_{i j} \leq \alpha_{i j}^{2} x_{i j}^{T} W_{i j}^{T} W_{i j} x_{i j}
$$

where $\alpha_{i j}$ is a positive number to be maximized and $W_{i j}$ is a full rank constant matrix; it can be set as an identity matrix in case that the information of uncertainty is unavailable. Pairwise subsystem (37) is robustly stabilizable with arbitrarily large basic coordinated controller degree $\alpha$ by the basic coordinated controller (21), a LMI problem that can be obtained as

$$
\begin{aligned}
& \operatorname{minimize} \quad \gamma_{i j} \\
& \text { subject to } Y_{i j}>0 \\
& {\left[\begin{array}{ccc}
A_{D i j} Y_{i j}+Y_{i j} A_{D i j}^{T}+B_{D i j} L_{i j}+L_{i j}^{T} B_{D i j}^{T} & A_{D i j} & Y_{i j} W_{i j}^{T} \\
A_{D i j}^{T} & -I & 0 \\
W_{i j}^{T} Y_{i j} & 0 & -\gamma_{i j} I
\end{array}\right]} \\
& <0 \text {, }
\end{aligned}
$$

where $L_{i j}=K_{D i j} Y_{i j}$ and $\gamma_{i j}=1 / \alpha_{i j}^{2}$. Further details of this robust control procedure can be found in [21, 22].

According to the permuted inclusion principle, the expanded system $\widetilde{\mathbf{S}}_{P}$ is supposed to contain those particular pairwise subsystems listed as following:

$$
\widetilde{\mathbf{S}}_{P}=\left\{\mathbf{S}_{12}, \mathbf{S}_{23}, \mathbf{S}_{13}, \mathbf{S}_{14}\right\}
$$

Then compose the coordinated controller of system $\widetilde{\mathbf{S}}_{P}$ by arranging the basic coordinated controllers of pairwise subsystems $\mathbf{S}_{12}, \mathbf{S}_{23}, \mathbf{S}_{13}$, and $\mathbf{S}_{14}$ in this exact recurrent reverse order,

$$
\begin{aligned}
& \widetilde{K}_{D}=\operatorname{blockdiag}\left(K_{D 12}, K_{D 23}, K_{D 13}, K_{D 14}\right) \\
&=\operatorname{blockdiag}\left(\left[\begin{array}{ll}
K_{11} & K_{12} \\
K_{21} & K_{22}
\end{array}\right],\left[\begin{array}{ll}
K_{22} & K_{23} \\
K_{32} & K_{33}
\end{array}\right],\right. \\
& {\left.\left[\begin{array}{ll}
K_{11} & K_{13} \\
K_{31} & K_{33}
\end{array}\right],\left[\begin{array}{ll}
K_{11} & K_{14} \\
K_{41} & K_{44}
\end{array}\right]\right) . }
\end{aligned}
$$

Choose the transformation matrices by (24)

$$
\begin{aligned}
& V=\operatorname{blockdiag}\left(\left[\begin{array}{lll}
I_{1}^{A} & I_{1}^{A} & I_{1}^{A}
\end{array}\right]^{T},\left[\begin{array}{ll}
I_{2}^{A} & I_{2}^{A}
\end{array}\right]^{T},\left[\begin{array}{ll}
I_{3}^{A} & I_{3}^{A}
\end{array}\right]^{T}, I_{4}^{A}\right) \\
& Q=\operatorname{blockdiag}\left(\frac{1}{3}\left[\begin{array}{lll}
I_{1}^{B} & I_{1}^{B} & I_{1}^{B}
\end{array}\right], \frac{1}{2}\left[\begin{array}{ll}
I_{2}^{B} & I_{2}^{B}
\end{array}\right], \frac{1}{2}\left[\begin{array}{ll}
I_{3}^{B} & I_{3}^{B}
\end{array}\right], I_{4}^{B}\right),
\end{aligned}
$$

and the permutation matrix $P_{A}$ can be constructed by (2); $P_{B}$ is in the same structure as $P_{A}$,

$$
P_{A}=\left[\begin{array}{cccccccc}
0 & 0 & 0 & 0 & 0 & 0 & I_{1}^{A} & 0 \\
0 & 0 & 0 & 0 & I_{1}^{A} & 0 & 0 & 0 \\
I_{1}^{A} & 0 & 0 & 0 & 0 & 0 & 0 & 0 \\
0 & I_{2}^{A} & 0 & 0 & 0 & 0 & 0 & 0 \\
0 & 0 & I_{2}^{A} & 0 & 0 & 0 & 0 & 0 \\
0 & 0 & 0 & I_{3}^{A} & 0 & 0 & 0 & 0 \\
0 & 0 & 0 & 0 & 0 & I_{3}^{A} & 0 & 0 \\
0 & 0 & 0 & 0 & 0 & 0 & 0 & I_{4}^{A}
\end{array}\right]
$$

where the dimensions of $I_{k}^{A}$ and $I_{k}^{B}$ are determined by the system state and control input vector, respectively. In this simulation example, $I_{k}^{A}=I_{6 \times 6}, I_{k}^{B}=1$.

Use (34) to construct the complementary matrix $\widetilde{M}_{K P}$ as follows:

$$
\widetilde{M}_{K P}=\left[\begin{array}{cccccccc}
0 & 0 & 0 & 0 & 0 & K_{13} & 0 & K_{14} \\
0 & 0 & 0 & K_{23} & 0 & 0 & 0 & 0 \\
K_{21} & 0 & 0 & 0 & 0 & 0 & 0 & 0 \\
0 & 0 & 0 & 0 & K_{31} & 0 & 0 & 0 \\
0 & K_{12} & 0 & 0 & 0 & 0 & 0 & K_{14} \\
0 & 0 & K_{32} & 0 & 0 & 0 & 0 & 0 \\
0 & K_{12} & 0 & 0 & 0 & K_{13} & 0 & 0 \\
0 & 0 & 0 & 0 & 0 & 0 & 0 & 0
\end{array}\right] .
$$

Finally, the coordinated controller of $\mathbf{S}$ can be contracted as

$$
\begin{aligned}
K & =\bar{K}=Q_{P}\left(\widetilde{K}_{D}+\widetilde{M}_{K P}\right) V_{P} \\
& =\left[\begin{array}{cccc}
K_{11} & K_{12} & K_{13} & K_{14} \\
K_{21} & K_{22} & K_{23} & 0 \\
K_{31} & K_{32} & K_{33} & 0 \\
K_{41} & 0 & 0 & K_{44}
\end{array}\right] .
\end{aligned}
$$

Figures 2 and 3 illustrate the frequency and tie-line power perturbations of the group of subsystems. The respond curves are very similar to those of [4-7].

\section{Conclusion}

This paper presents a theoretical study of the pairwise decomposition, which can be seen as a reverse modality of this methodology. The proposed approach is able to coordinated the interconnected system with uncertainties, and it can achieve high quality control performance as well. Moreover, this process is convenient for a group of interconnected subsystems without a superposition-form overall system model, which is in the case that only local information is available. Further research is ongoing, and one task is to determine the structure of system $\overline{\mathbf{S}}$ as an estimator of the original system S. For this purpose, an update law which can fit the features of pairwise decomposition is needed as well as a calculation framework to deal with the structure perturbations effectively enough. The proposed approach can also motivate the application of pairwise decomposition to a nonlinear time-variant system. 


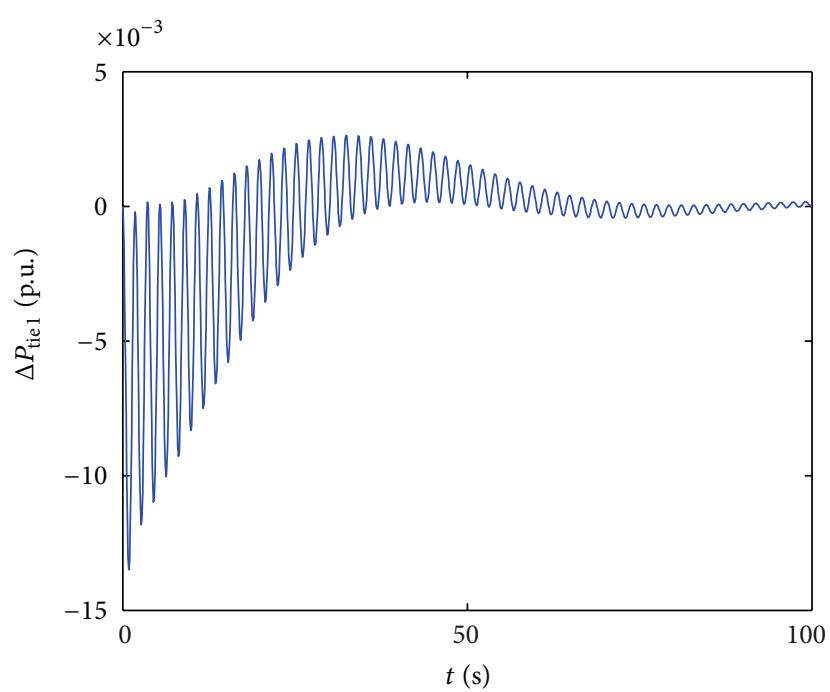

(a)

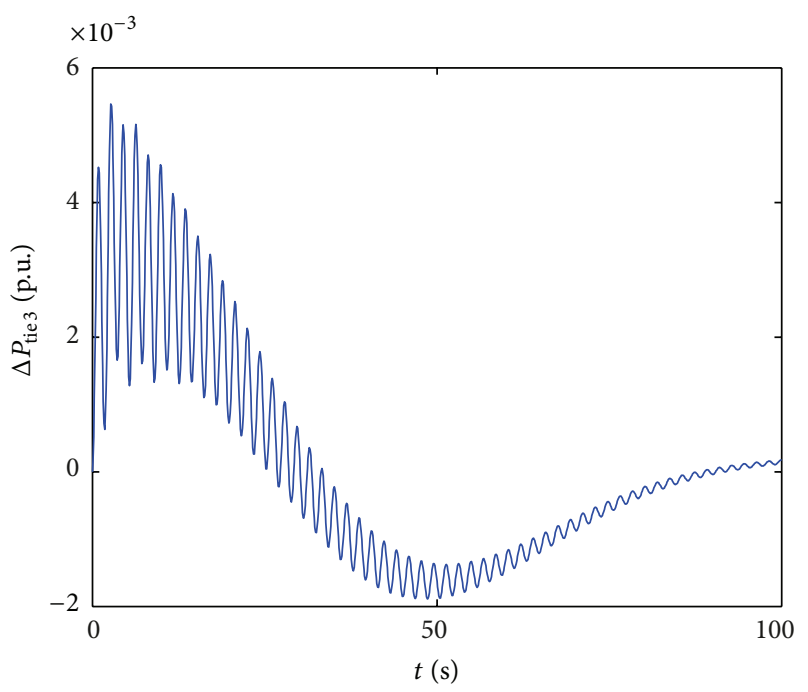

(c)

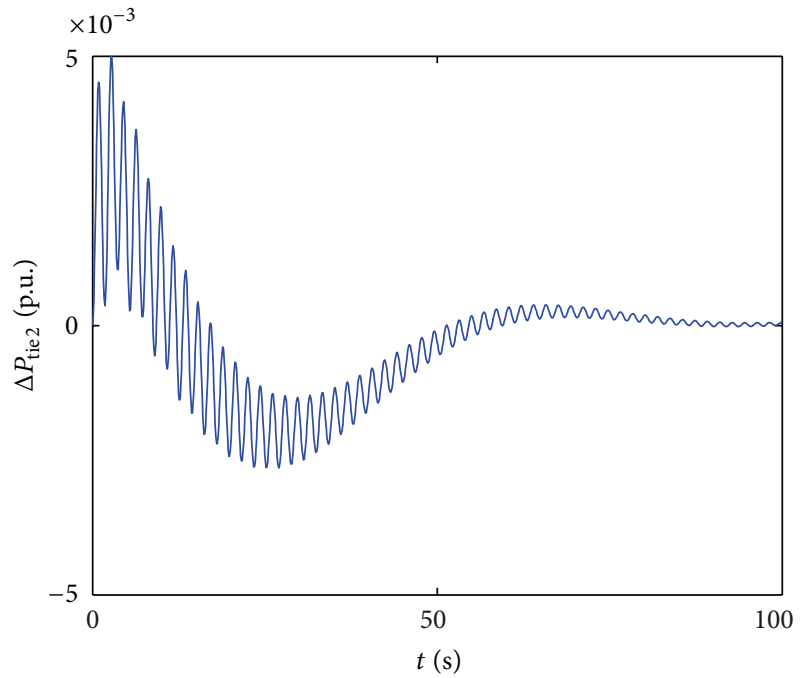

(b)

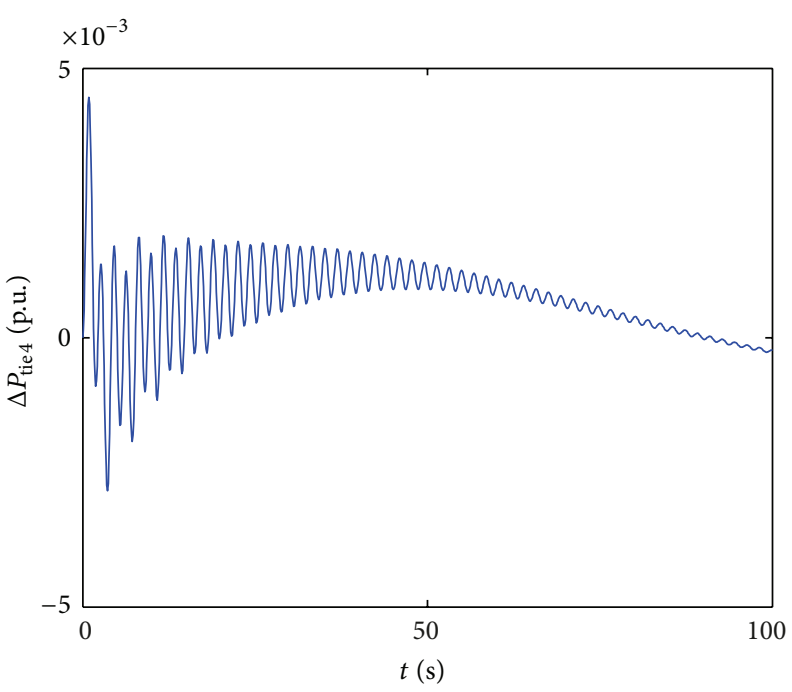

(d)

FIGURE 3: Deviations of tie-line power among the power system.

\section{Acknowledgment}

This research reported herein was supported by the NSF of China under Grant no. 60874017.

\section{References}

[1] M. Ikeda and D. D. Šiljak, "Overlapping decompositions, expansions and contractions of dynamic systems," Large Scale Systems, vol. 1, no. 1, pp. 29-38, 1980.

[2] M. Ikeda, D. D. Šiljak, and D. E. White, "An inclusion principle for dynamic systems," IEEE Transactions on Automatic Control, vol. 29, no. 3, pp. 244-249, 1984.

[3] D. D. Šiljak, Decentralized Control of Complex Systems, Academic Press, New York, NY, USA, 1991.

[4] X.-B. Chen and S. S. Stanković, "Decomposition and decentralized control of systems with multi-overlapping structure," Automatica, vol. 41, no. 10, pp. 1765-1772, 2005.
[5] X.-B. Chen and S. S. Stanković, "Overlapping decentralized approach to automation generation control of multi-area power systems," International Journal of Control, vol. 80, no. 3, pp. 386402, 2007.

[6] X.-B. Chen, W.-B. Xu, T.-Y. Huang, X.-Y. Ouyang, and S. S. Stanković, "Pair-wise decomposition and coordinated control of complex systems," Information Sciences, vol. 185, no. 1, pp. 7899, 2012.

[7] X.-B. Chen, System Inclusion Principle and Its Application, Science Press, Beijing, PRC, 2012, (Chinese).

[8] X.-B. Chen and C. Ma, "Coordinated control of a four-area power system under structural perturbation," in Proceedings of the 9th Asian Control Conference, Istanbul, Turkey, 2013.

[9] D. M. Stipanović, G. Inalhan, R. Teo, and C. J. Tomlin, "Decentralized overlapping control of a formation of unmanned aerial vehicles," Automatica, vol. 40, no. 8, pp. 1285-1296, 2004. 
[10] F. Palacios-Quiñonero, J. Rodellar, and J. M. Rossell, "Sequential design of multi-overlapping controllers for longitudinal multioverlapping systems," Applied Mathematics and Computation, vol. 217, no. 3, pp. 1170-1183, 2010.

[11] F. Palacios-Quiñonero, J. M. Rossell, and H. R. Karimi, "Semidecentralized strategies in structuralm vibration control," Modeling, Identification and Control, vol. 32, no. 2, pp. 57-77, 2011.

[12] F. Palacios-Quiñonero, J. Rubió-Massegú, J. M. Rossell et al., "Discretetime multioverlapping controller design for structural vibration control of tall buildings under seismic excitation," Mathematical Problems in Engineering, vol. 2012, Article ID 636878, 20 pages, 2012.

[13] M. E. Sezer and D. D. Šiljak, "Validation of reduced-order models for control systems design," Journal of Guidance, Control, and Dynamics, vol. 5, no. 5, pp. 430-437, 1982.

[14] M. Ikeda and D. D. Šiljak, "Overlapping decentralized control with input, state, and output inclusion," Control Theory and Advanced Technology, vol. 2, no. 2, pp. 155-172, 1986.

[15] S. S. Stanković, X.-B. Chen, M. R. Matausek et al., "Stochastic inclusion principle applied to decentralized overlapping suboptimal LQG control," International Journal of Control, vol. 72, no. 3, pp. 276-288, 1999.

[16] D. D. Šiljak, "Dynamic graphs," Nonlinear Analysis: Hybrid Systems, vol. 2, no. 2, pp. 544-567, 2008.

[17] S. S. Stanković and D. D. Šiljak, "Sequential LQG optimization of hierarchically structured systems," Automatica, vol. 25, no. 4, pp. 545-559, 1989.

[18] S. S. Stanković and D. D. Šiljak, "Inclusion principle for linear time-varying systems," SIAM Journal on Control and Optimization, vol. 42, no. 1, pp. 321-341, 2003.

[19] C. S. Chang and W. Fu, "Area load frequency control using fuzzy gain scheduling of PI controllers," Electric Power Systems Research, vol. 42, no. 2, pp. 145-152, 1997.

[20] H. L. Zeynelgil, A. Demiroren, and N. S. Sengor, “The application of ANN technique to automatic generation control for multi-area power system," International Journal of Electrical Power and Energy Systems, vol. 24, no. 5, pp. 345-354, 2002.

[21] S. Boyd, L. El Ghaoui, E. Feron et al., Linear Matrix Inequalities in System and Control Theory, SIAM, Philadelphia, PA, USA, 1994.

[22] D. D. Šiljak and D. M. Stipanović, "Robust stabilization of nonlinear systems: the LMI approach," Mathematical Problems in Engineering, vol. 6, no. 5, pp. 461-493, 2000. 


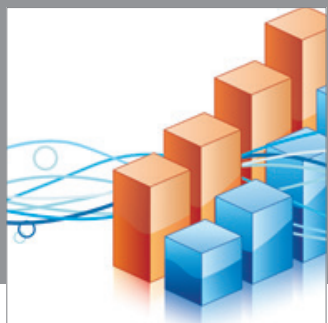

Advances in

Operations Research

mansans

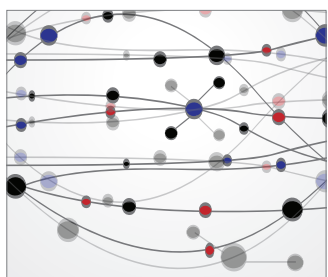

The Scientific World Journal
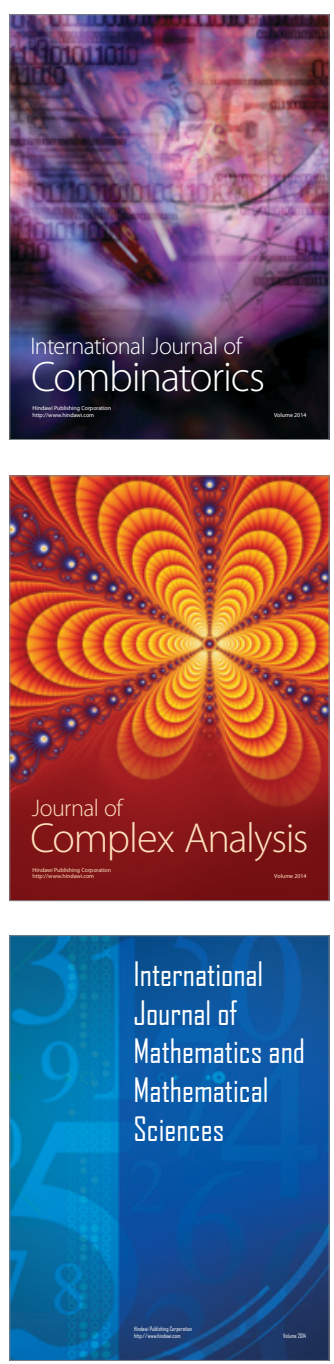
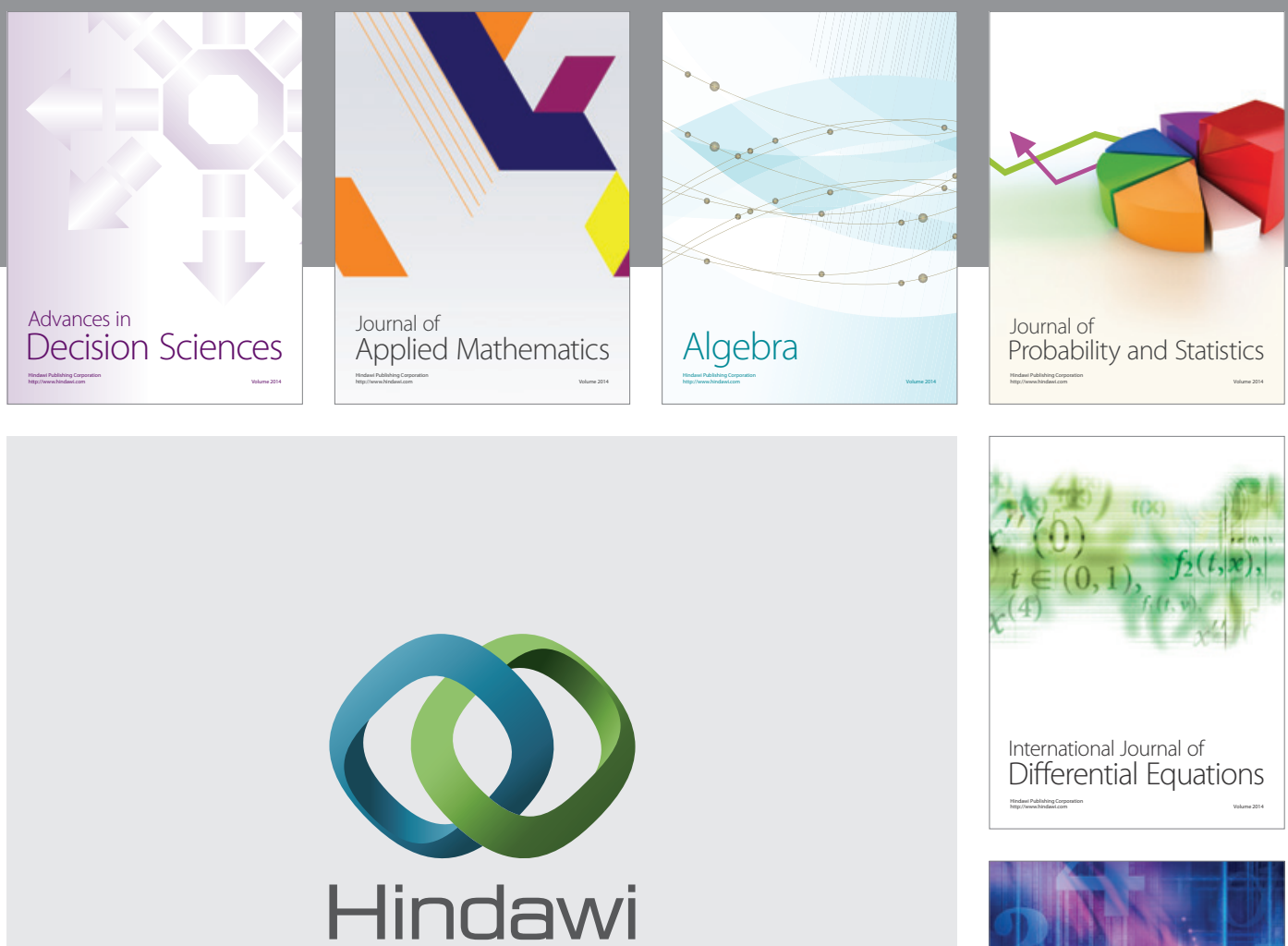

Submit your manuscripts at http://www.hindawi.com
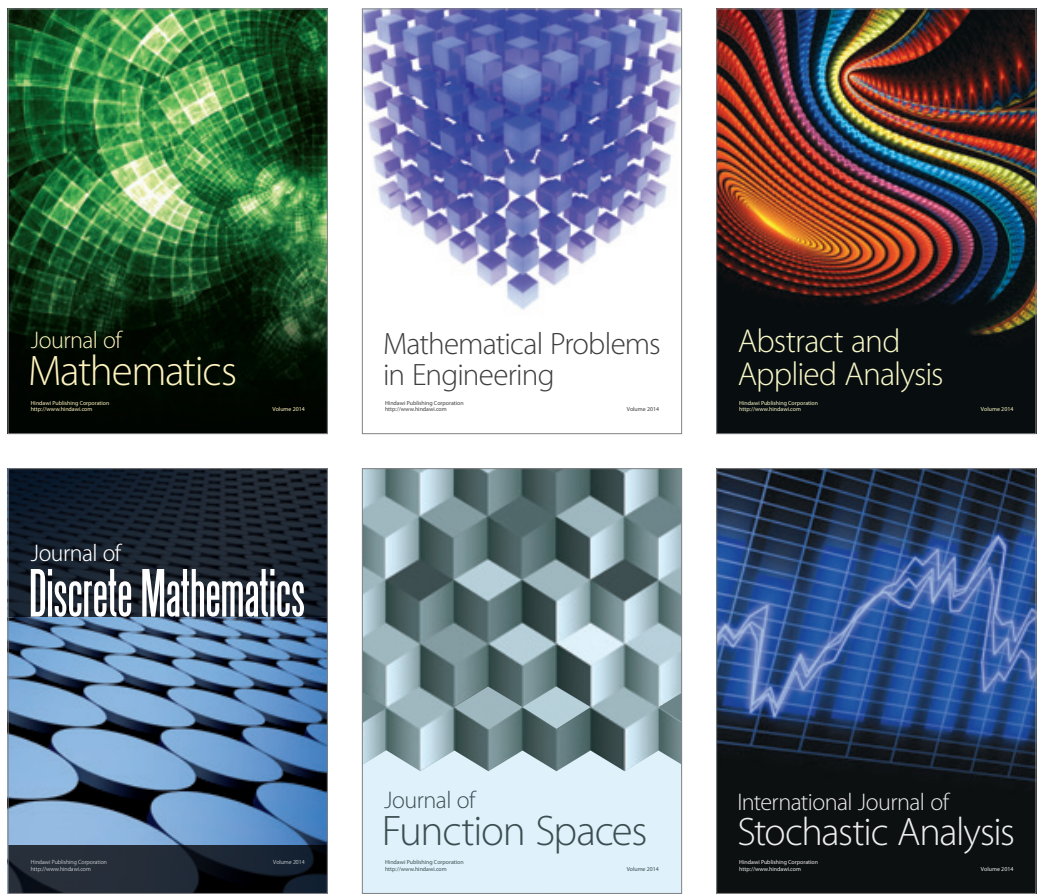

Journal of

Function Spaces

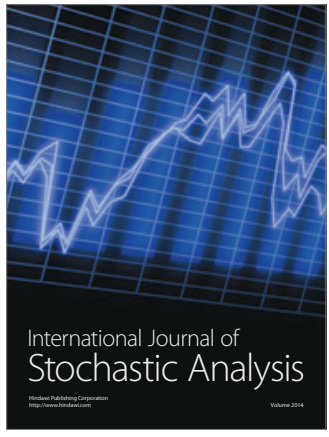

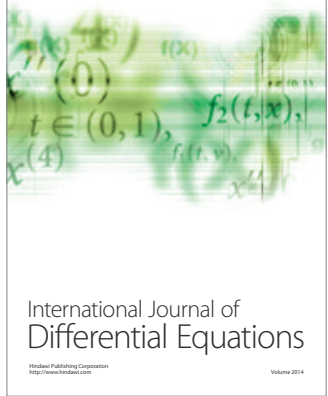
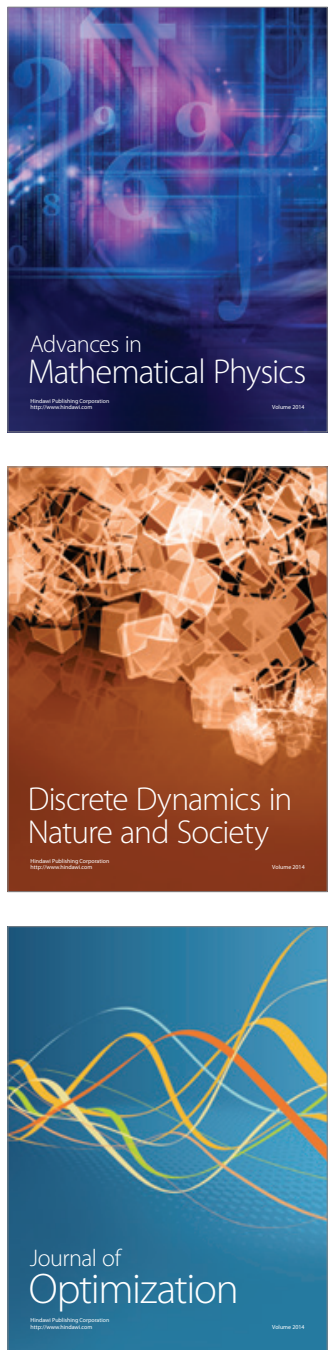